\title{
Application of Data Bus-Based Software Architecture in Wind Turbine Control Software
}

\author{
Meiyu Cui and Yongjun Qie
}

\begin{abstract}
In this paper, a data bus-based software architecture is designed to provide standardized, transparent software interfaces, to reduce coupling between software modules, and shield the complexity of operating system for synchronous reactive system software design. First of all, based on synchronous theory, a data bus-based software architecture is designed. And then, common data space is designated, it is open to all application layer software module, each application layer software module can directly access the common data space. At the same time, a data protection mechanism has been established to ensure that every data has single source and all data are updated uniformly. Finally, the software architecture is applied in wind turbine control software, and the wind turbine control software architecture is reconstructed.
\end{abstract}

M. Cui · Y. Qie (ه)

Tsinghua University, Haidian District, Beijing, China

e-mail: xiyj19@mail.tsinghua.edu.cn 